\title{
In Situ Observation of Gold Nanoparticles Self-assembly at the Solid-Liquid Interface Using Liquid-Phase STEM
}

\author{
Arixin Bo, Björn Kuttich, Tobias Kraus and Niels de Jonge \\ INM - Leibniz Institute for New Materials, Saarbrücken, Germany
}

Self-assembly for uniform nanostructures has been an active research area posing significant technological importance to the bottom-up construction of nanoscale devices. By tuning variables such as ligand coating, particle shapes, solvent, and preparation method, it is possible to obtain desired self-assembly patterns [1]. However, continuous research attempts are required to fully understand each of the nanoscale selfassembly steps where it faces numerous challenges. Herein, dynamic self-assembly behavior is one of the challenges that draws great scientific and technological interests.

Observation of nanostructure assembly at the solid-liquid interface can be achieved by applying liquid phase scanning transmission electron microscopy (LP-STEM) [2]. Imaging in liquid excludes disruptions from the liquid surface tension or assembled feature deposition from the liquid-gas interface. In this work, we captured and analyzed the assembly pattern formation of oleylamine capped gold nanoparticles (AuNPs) at the plasma-activated SiN surface (SiN-P) in toluene solution. Due to the strong polarity of the SiN-P surface, the colloid AuNPs are polarized and attracted to the surface forming densely packed hexagon structures [3]. Combining LP-STEM with a microfluidic syringe pump (incoming flow rate at 2 $\mu \mathrm{L} / \mathrm{min}$ ), we captured in situ AuNPs assembly behavior where the colloid deposition occurred primarily at the edge of the nucleated assembly pattern instead of random deposition (Fig. 1). In this non-polar organic solution, we speculated that the substrate-particle-liquid interface exerts highest of surface energies and attracts AuNPs from the colloid system. Such surface energy preference resembles that of a crystal growth where atoms proliferate at the step edges and the rate is dependent on the density of kink sites [4].

The polarization-induced interactions of the assembled particles are strong enough to stabilize the assembly on the surface against Brownian motion caused by random collisions with solvent molecules. Convective liquid motion, for example caused by heating, was strong enough to disrupt the assembly, however. We observed drastic changes and rearrangement of the assembled clusters on SiN-P (Fig. 2). This suggests the assembled AuNPs could relocate and likely merge with their neighbors when attracted. Capturing assembly processes under different magnifications, the changing electron dose could affect the assembly behavior. Based on the assembly systems, the degree of electron beam effect requires further explorations. Using LP-STEM, dynamic self-assembly studies provide direct insights for the nextgeneration production designs and serve as strong support in the relating theoretical investigations [5].
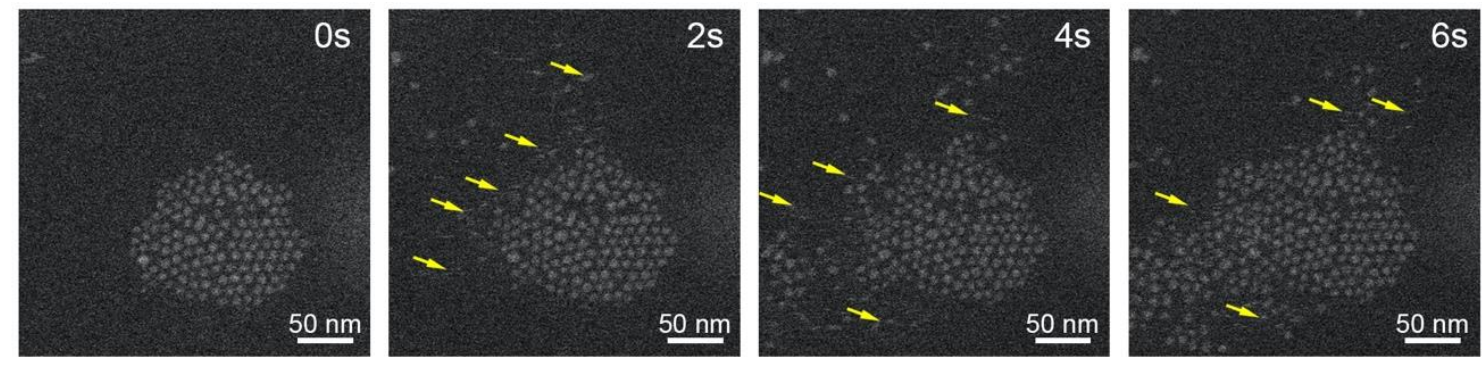

Figure 1. LP-STEM images depicting in situ deposition and self-assembly of AuNP-oleylamine in toluene on SiN-Plasma surface. The arrows point to the incoming AuNPs. 

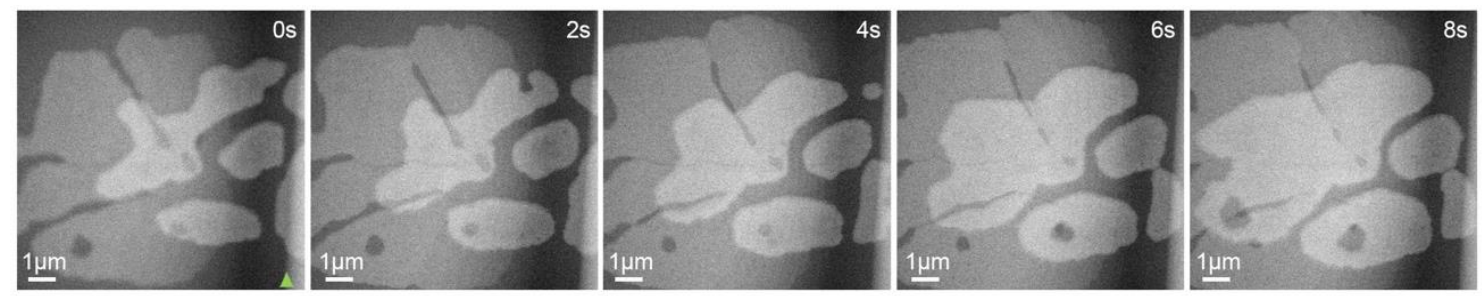

Figure 2. LP-STEM images showing the movement of assembled AuNP clusters in toluene caused by liquid turbulence from heating $\left(45^{\circ} \mathrm{C}\right.$ hold). The green marker indicates the position of the $\mathrm{SiN}$ window edge, along which the assembled AuNP area reduced.

\section{References}

[1] M C Weidman et al., Nat Mater, 15 (2016) p. 775.

[2] N de Jonge and F M Ross, Nat Nanotechnol, 6 (2011) p. 695.

[3] E Cepeda-Perez et al., Sci Adv, 6 (2020) p. 1404.

[4] M N Joswiak et al., Cryst Growth Des, 18 (2018) p. 723.

[5] The authors thank Arzt for his support through INM. 